\title{
Estimation of Wave Movement Using Gauss-Markov Estimation
}

\author{
Yoshiharu Koya \\ Dept. of Electronic Engineering, Kobe City College of Technology \\ 8-3, Gakuen Higashimachi, Nishi-ku, Kobe, Hyogo 651-2194, Japan \\ E-mail: y-koya@kobe-kosen.ac.jp
}

\begin{abstract}
The number of web cameras installed along shorelines has increased significantly in recent years. Unfortunately, these cameras are overwhelmingly passive in their application: they monitor ocean conditions but do nothing to detect threats and alert other systems. This constitutes a lost opportunity, especially in light of recent hurricane and tsunami disasters. Though such threats can be predicted by meteorological or seismological agencies, localized predictions of the size and arrival times of large waves have not been feasible. Hence, there is a need for a broadly deployable system for detecting wave dynamics.

In this paper, we propose such a system. Using standard video footage from web cameras, our system can detect local formation of large waves and thereby reduce their threat potential. Unlike previously proposed wave detection systems based on imaging, such as conventional block matching methods, ours does not require particularly high resolution or stereography, nor is it computationally complex. Based purely on calculating the phase difference between consecutive images of a wave using Fourier transform, our system can detect the displacement of waves with high accuracy.
\end{abstract}

\section{Introduction}

Typically, we use block matching or optical flow estimation to approximate the displacement of motion in a moving image. These approaches may or may not give good results, even when the target domain has a strong characteristic feature for extraction. In the conventional method, for instance, if we extract the domain of white waves and perform block matching, we can accurately estimate the displacement of waves, but if we perform optical flow analysis on this same domain, our estimation fails, since the extracted white waves don't exhibit a useful change in brightness. In either case, the fundamental problem is that white waves are not a common formation, and ocean water otherwise tends to be very uniform in color. In short, there is no characteristic domain to which we can reliably apply block matching and optical flow estimation.

To address this problem, we propose a new method using Fourier transform on the entire image of a set of waves, thus avoiding the need to extract a characteristic domain.
Similar approaches using the Fourier phase correlation method or the phase-only correlation method [1][2] have been proposed, but these require the additional and expensive step of computing the correlation in the phase after having performed the Fourier transform. Though such methods are applicable even to images in which there are few changes of brightness (e.g., those that only require translation, such as biometric authentication), given our focus on the problem of displacement of waves, they are of little value to us. Furthermore, because these methods require three separate Fourier transforms, the computational complexity is too great for real-time calculation. Therefore, in this study, we estimate the quantity of movement of the wave to reduce calculation time.

In our method, we perform Fourier transforms and compute the phase in each frequency for every frame. Then, based on the phase difference between frames, we estimate the displacement of movement. Note that very different movement quantities may be computed at different frequencies, so it is necessary to estimate the overall displacement of a wave. In this study, we based our estimate on the weight of the error term in the Gauss-Markov estimate [3][4].

We computed the displacement of the wave in one dimension. This computation is intended to support accurate prediction of when a threatening wave formation will arrive at the local shoreline.

\section{Target data}

The image in figure 1 is of offshore waves at Hinomisaki in Wakayama prefecture in Japan. It was captured at a rate of 30 frames per second (fps) and a resolution of $1800 \times$ 1800 using the CASIO EX-FH25 video camera.

In figure 2, we see the wave brightness for $\mathrm{x}=900$ of the given image. Though we can measure the full RGB signal in the image, we expect that the brightness of the green and blue channels will change slightly, given that they are the dominant colors in the image. In contrast, the brightness of the red channel exhibits considerable change, so it offers a strong characteristic domain for estimating the displacement of a wave. 


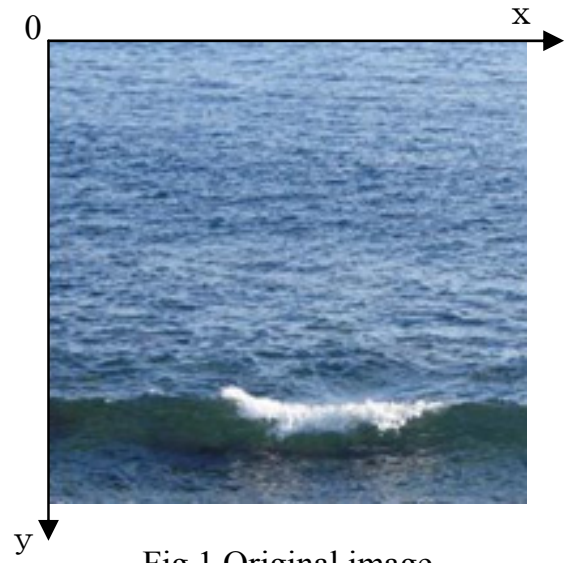

Fig.1 Original image $(1800 \times 1800)$

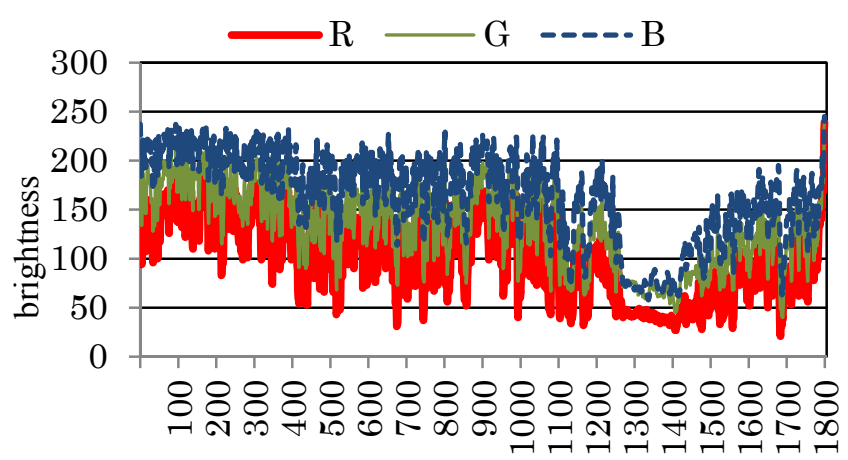

Direction of $y$

Fig.2 Brightness for $\mathrm{x}=900$ in figure 1

\section{Estimation method}

To apply our technique, we obtain continuously recorded images of a section of open water. We approximate the wave signal in these images using a Fourier transform, and then extract the phase difference between successive frames to estimate wave displacement. More formally, the discrete Fourier transforms $\mathrm{G}_{1}$ and $\mathrm{G}_{2}$ of a pair of sampled images $g_{1}$ and $g_{2}$ are computed, and the phase difference $\mathrm{e}^{\mathrm{j}\left(\phi_{1}-\phi_{2}\right)}$ is obtained for each spatial frequency, where $\varnothing=\emptyset_{1}-\emptyset_{2}$ denotes the phase.

\subsection{Fourier transform}

We give the equation for our Fourier transform in (3.1) and the initial phase $\varphi$ of this equation in (3.2). Accordingly, we compute the difference $(\Delta \varphi)$ of $\varphi$ between consecutive frames. When the interval is short, a linear phase characteristic is established if the wave moves without the whole wave changing form, yielding a constant value for the difference $(\Delta \varphi)$ across all frequencies.
However, a wave pattern will not necessarily maintain its form, and new occlusions may prevent the top and bottom of the wave from remaining within the observable domain. Therefore, we compute the difference $\Delta \varphi$ across all frequencies.

$$
\begin{gathered}
\mathrm{g}[\mathrm{y}]=\sum_{\mathrm{k}=0}^{\mathrm{N}-1}\left\{\mathrm{a}[\mathrm{k}] \cos \left(\frac{2 \pi \mathrm{y}}{\mathrm{N}}\right)+\mathrm{b}[\mathrm{k}] \sin \left(\frac{2 \pi \mathrm{yk}}{\mathrm{N}}\right)\right\} \\
\mathrm{N}: 1800(\text { size of } \mathrm{y}) \quad \mathrm{k}: \text { frequency } \\
\mathrm{a} \sin \alpha+\mathrm{b} \cos \alpha=\sqrt{\mathrm{a}^{2}+\mathrm{b}^{2}} \sin (\alpha+\emptyset) \\
\left(\text { note: } \tan \emptyset=\frac{\mathrm{b}}{\mathrm{a}}, \emptyset: \text { phase }\right)
\end{gathered}
$$

\subsection{Displacement a wave by phase estimation}

Next, we estimate the brightness waveform for any given $\mathrm{x}$. When this brightness waveform undergoes Fourier transform, we calculate the phase from base to the $900^{\text {th }}$ frequency for consecutive frames and compute the difference between them. The phase difference corresponding to the number of pixels from a phase difference using 1,800 pixels is $2 \pi$. Thus, we can find changing pixels in each frame, but we cannot determine the exact displacement per pixel. Figure 3 shows the displacement of a wave estimated by phase difference.

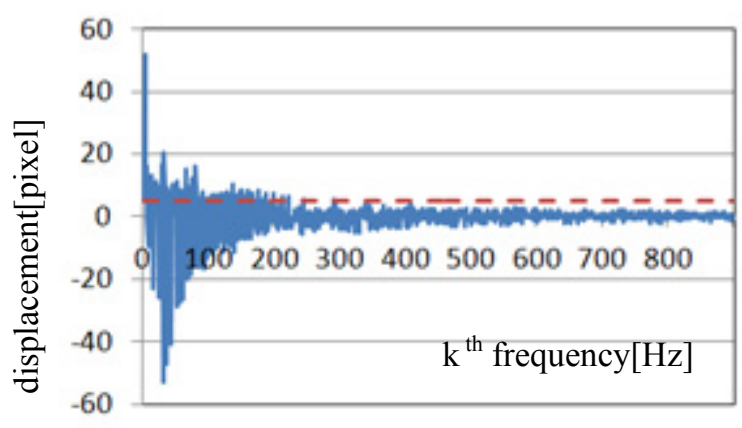

Fig.3 Displacement of movement for $\mathrm{x}=900$

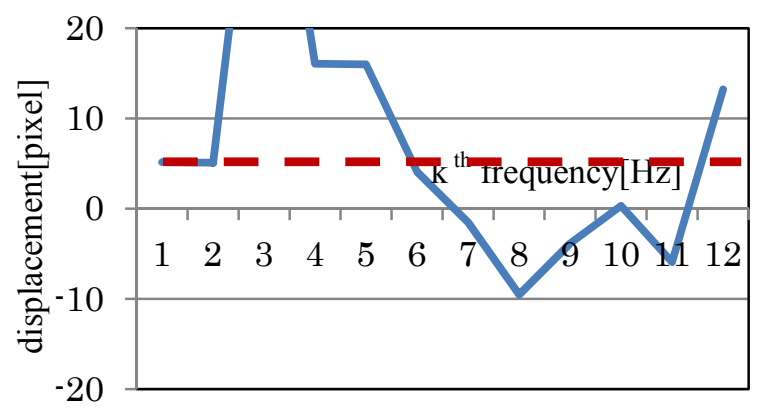

Fig.4 Displacement of movement for $\mathrm{x}=900$ (Low frequency) 
Note that for reference, we also estimated wave displacement using the aforementioned block matching technique on white waves. This reference is indicated by the red dotted lines in figures 3 and 4.

In this result, the high-frequency component becomes smaller than the targeted value. The one-period length for each frequency is smaller than the displacement in the wave, indicating that the correct displacement has not been estimated.

In addition, there appears to be an extra factor involved at higher frequencies, since neither end of the wave pattern is uniform in brightness. Thus, when we calculate the displacement of a wave, it is necessary to use only a low frequency and to restrict our estimate to this lower band, as in figure 4 . If the frequency is too low, i.e., near the base wave frequency, data for the bottom end disappears and data for the upper end increases.

In figure 4, we see good results in the sixth frequency, and can confidently base our estimate on that frequency. To calculate the final displacement, however, we must assign a weighting to each frequency, since results from the sixth frequency alone may bias our estimate.

\subsection{Weighting by normal distribution}

To assign appropriate weights to each frequency, we first establish the sixth frequency as the center of a normal distribution, thereby suppressing the influence of high frequencies. The normal distribution is given in equation (3.3), where $\mathrm{k}$ is frequency.

$$
f(k)=\frac{1}{\sqrt{2 \pi \sigma^{2}}} \exp \left(-\frac{(k-\mu)^{2}}{2 \sigma^{2}}\right)
$$

To use equation (3.3) we need to set a suitable $\sigma$ and $\mu$. Having determined that the sixth frequency is the most reliable, we set $\mu$ as 6 . Since over $99 \%$ of data is secured if we used $3 \sigma$ in normal distribution, we give $3 \sigma$ a provisional value of 1 . In other words, we decide on a range of $3 \sigma$ for 5 from the basic wave to the sixth frequency. A graph of the resulting weighting is shown in figure 5. Note that the weighting coefficients differ according to the size of the image, so the sixth frequency may not receive the largest weighting.

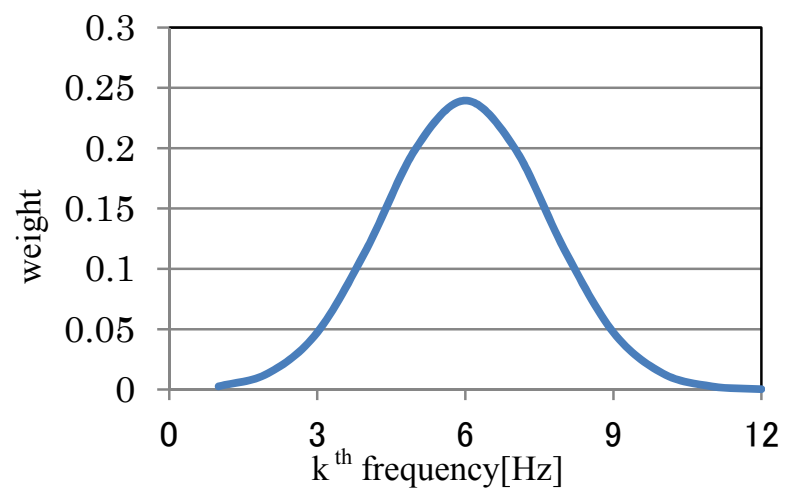

Fig.5 Weight by normal distribution

\subsection{Movement by Gauss-Markov Estimation}

Given the weighting calculated by the normal distribution above, we know that an error on the simple weighted average was retained, since all the data together did not add up to $100 \%$. To correct this error, we estimated the final movement using a reciprocal of the weight of each frequency for the error term of the Gauss-Markov estimation. This estimation is given in (3.5), and its displacement represents our final value for the displacement of wave movement.

$$
\begin{gathered}
\emptyset=\mathrm{Z} \alpha+\varepsilon \quad \cdots(3.4) \\
\phi=\left[\begin{array}{c}
\emptyset_{1} \\
\vdots \\
\emptyset_{15}
\end{array}\right], \quad \alpha=[\alpha], \quad \varepsilon=\left[\begin{array}{c}
\varepsilon_{1} \\
\vdots \\
\varepsilon_{15}
\end{array}\right], Z^{=}\left[\begin{array}{c}
1 \\
\vdots \\
1
\end{array}\right] \\
\alpha=\left(\mathrm{Z}^{\mathrm{T}}\left(\varepsilon \varepsilon^{\mathrm{T}}\right)^{-1} \mathrm{Z}\right)^{-1} \mathrm{Z}^{\mathrm{T}}\left(\varepsilon \varepsilon^{\mathrm{T}}\right)^{-1} \varphi \quad \cdots(3.5) \\
\phi: \text { phase difference (measurements) } \quad \mathbf{Z} \text { :base } \\
\alpha \text { :coefficient } \quad \text { : error }
\end{gathered}
$$

We show the results of this Gauss-Markov estimation in table 1 . Note that the target value has no decimal place because it is calculated per-pixel with block matching. We considered using a coefficient of correlation, but chose not to in this case because there was a part of the signal that flamed out, causing the target range to shift.

Table 1 Movement displacement

\begin{tabular}{|c|c|c|}
\hline method & $\begin{array}{c}\text { Block matching } \\
\text { (target value) }\end{array}$ & $\begin{array}{c}\text { Gauss-Markov } \\
\text { (estimation value) }\end{array}$ \\
\hline value[pixel] & 5 & 5.98 \\
\hline
\end{tabular}

Though the value of our estimate is very close to the target value, we must acknowledge that moving the ROI (region of interest) for block matching only one pixel will produce an error of \pm 0.5 pixels. This error is significant, but since the target value is used only as a reference, and not as a true measure of displacement of a wave, we find it tolerable.

\section{Examination}

For the tests above, we established the sixth frequency as our center, but overall we experimented with three different center frequencies: the basic wave frequency, the sixth frequency, and the twelfth frequency. We estimated the displacement of a wave using data for $\mathrm{x}=300$ and $\mathrm{x}=$ 1000 to consider whether this weighting was suitable, as seen in figures 6 and 7. Note that in either case, if the wave pattern is detected between the fifth and seventh frequencies, we get a value to the target displacement value of 5 , and so we conclude that it is best to set the center of the normal distribution to 6 . 


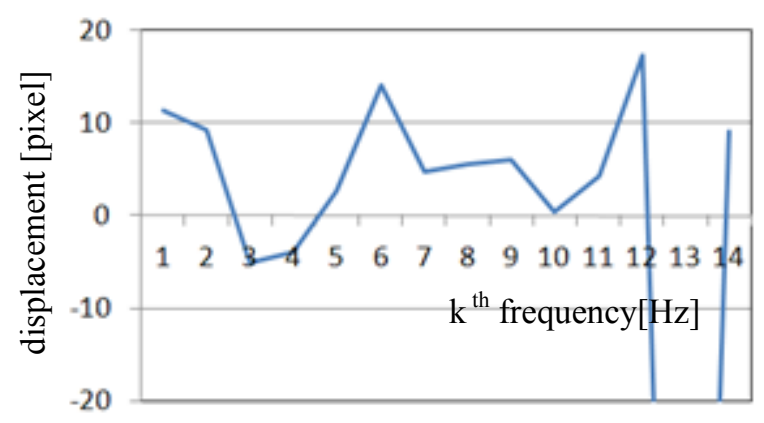

Fig.6 Displacement of movement for $\mathrm{x}=300$ (Low frequency)

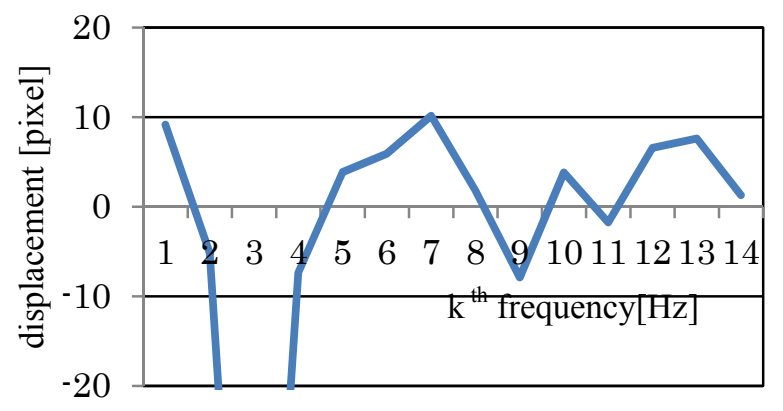

Fig.7 Displacement of movement for $\mathrm{x}=1000$ (Low frequency)

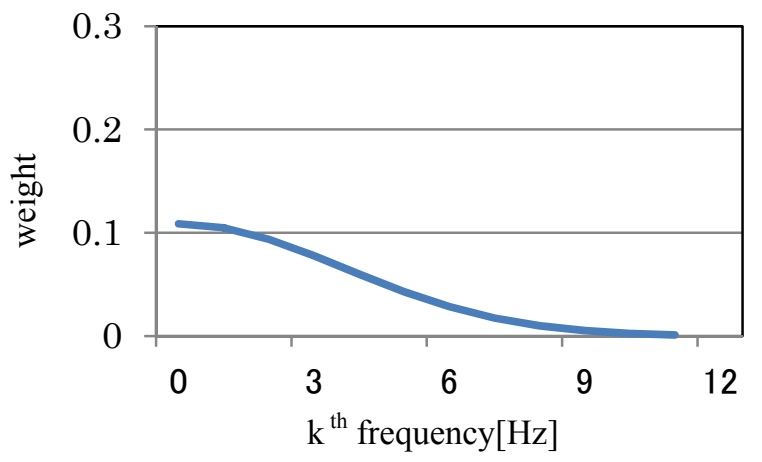

Fig.8 Weight by normal distribution centered at base frequency

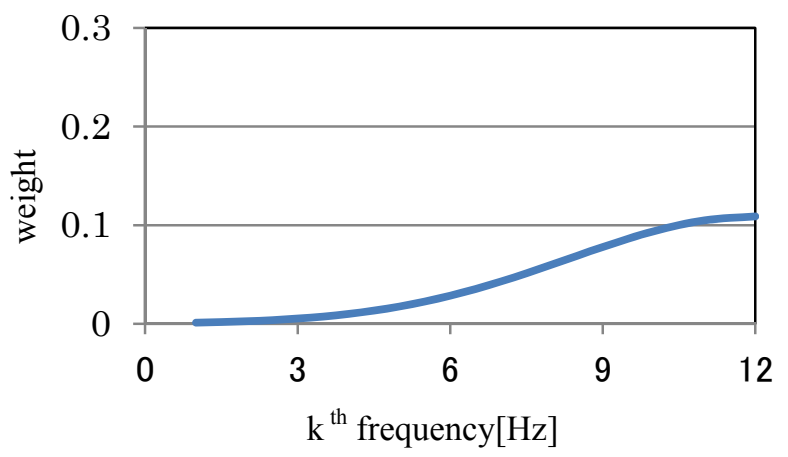

Fig.9 Weight by normal distribution centered at the $12^{\text {th }}$ frequency
In figures 8 and 9, we show the results of shifting the center of the weight distribution to the base frequency and the $12^{\text {th }}$ frequency, respectively. Note that with the center at the $12^{\text {th }}$ frequency, we could make use of higher frequencies, but we chose not to do so in the interest of comparison with other data. Our estimation results using these different weight distributions are given in table 2 .

Table 2 Displacement of movement by difference of weight

\begin{tabular}{|c|c|c|c|}
\hline \multicolumn{4}{|c|}{ weight } \\
\hline $\mathrm{x}=300$ & $\begin{array}{c}\text { First } \\
\text { frequency }\end{array}$ & $\begin{array}{c}6^{\text {th }} \\
\text { frequency }\end{array}$ & $\begin{array}{c}12^{\text {th }} \\
\text { frequency }\end{array}$ \\
\hline $\mathrm{x}=900$ & 4.70 & 6.66 & 7.49 \\
\hline $\mathrm{x}=1000$ & -15.49 & 5.98 & 1.02 \\
\hline
\end{tabular}

As is clear from the table, changing the center of the normal distribution leads to a substantial difference in the resulting estimate. However, when using the sixth frequency as our center, our estimate remains close to the target value, even if we use a wave signal for any given $\mathrm{x}$.

We compared the estimated amount of wave movement with that obtained using the block matching method. We used block matching to estimate the movement of a white wave domain. We show this result in figure 10. It is clear that the amount of rightward movement can be accurately estimated using the proposed method.

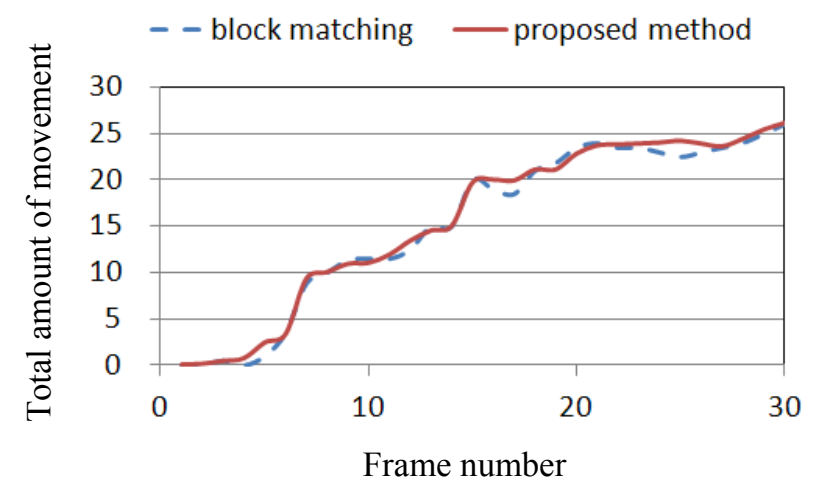

Fig.10 Estimation result

\section{Conclusions}

Our method of estimating wave displacement based on phase difference of the Fourier transform displacement produces reasonably good results with relatively few calculations. However, the displacement of constant movement is not estimated all in frequency, and different values are calculated. Thus, we did not restrict ourselves to one phase, and computed the displacement of a wave for multiple data inputs using Gauss-Markov estimation. Furthermore, because frequencies are not reliable to the same degree, we had to correct for the reliability of each frequency using a normal distribution for the error term of the Gauss-Markov estimation. 
Normally, it is necessary to use a matrix calculation with Gauss-Markov estimation, but since the parameter we are finding is a quantity of movement, we can dispense with matrix calculation altogether, significantly reducing calculation time. As a result, we can achieve nearly real-time calculation speeds for a 30fps system, a key benchmark for processing data streamed from the real world. Speed can be further improved by eliminating frames when the wave pattern does not change significantly.

To further test the reliability of our method, we intend to use data streamed from cameras actually deployed at various shorelines, rather than data captured by the author using a personally selected camera. The Ministry of Land, Infrastructure and Transport Himeji already supplies wave data, but the lack of white wave footage made proper evaluation implausible. To properly test our system against more raw data, we will have to find some other means (e.g., sonar) of objectively measuring the movement of monitored waves.

\section{Acknowledgments}

The authors would like to thank the anonymous reviewers for their constructive comments and suggestions. This work was supported in part by the Ministry of Land, Infrastructure and Transport, Himeji rivers national highway office.

\section{References}

[1] C.D. Kuglin and D.C. Hines, "The phase correlation image alignment method" in Proc. Int. Conf. Cybernetics and Society, pp. 163-165, Sept. 1975

[2] I. Izumi and K. Hitoshi, "Phase scrambling for POC between visually protected images," D-11-17 p. 71, The Institute of Electronics, Information and Communication Engineers (2009)

[3] H. Drygas, "Lecture notes in operations research and mathematical systems," No. 40, pp. 1-7, Springer (1970)

[4] Y. Koya and I. Mizoshiri, "Border detection on common carotid artery using Gauss-Markov estimation," T. IEEJ Japan, Vol. 124-C, No. 10, pp. 2051-2056 (2004) 